\title{
Why We Should Move from Reductionism and Embrace a Network Approach to \\ Parental Burnout
}

\author{
M. Annelise Blanchard ${ }^{1}$ and Alexandre Heeren ${ }^{1,2}$ \\ ${ }^{1}$ Psychological Sciences Research Institute, UCLouvain, Belgium \\ ${ }^{2}$ Institute of Neuroscience, UCLouvain, Belgium
}

In press in New Directions for Child and Adolescent Development

This is a preprint (version October 5, 2020) of a manuscript that has been peer-reviewed and accepted for publication in New Directions for Child and Adolescent Development. This paper is not the copy of record and may not exactly replicate the final, authoritative version of the article as published in New Directions for Child and Adolescent Development. The final article will be available, upon publication, via its DOI.

\author{
Author Note \\ M. Annelise Blanchard (D) https://orcid.org/0000-0002-9605-7022 \\ Alexandre Heeren (D) https://orcid.org/0000-0003-0553-6149 \\ We have no conflict of interest to disclose. \\ Correspondence concerning this article should be addressed to M. Annelise Blanchard. \\ Psychological Sciences Research Institute, Université Catholique de Louvain, Place du \\ Cardinal Mercier, 10, B-1348, Louvain-la-Neuve, Belgium. E-mail: \\ marie.blanchard@uclouvain.be
}




\begin{abstract}
Network science has allowed varied scientific fields to investigate and visualize complex relations between many variables, and psychology research has begun to adopt a network perspective. In this paper, we consider how leaving behind reductionist approaches and instead embracing a network perspective can advance the field of parental burnout. Although research into parental burnout is in its early stages, we argue that a network approach to parental burnout could set up the scene for radically new vistas in parental burnout research. We claim that such an approach can allow simultaneous investigations (and clear visualizations) of many variables related to parental burnout and their interactions, integrates smoothly with prior family systems theories, and prioritizes dynamic research questions. We likewise discuss potential future clinical applications, such as interventions targeting central nodes and treatment personalized to a specific family's network system. We also review practical considerations, limitations, and future directions for researchers interested in applying a network approach to parental burnout research.
\end{abstract}

Keywords: network analysis, parental burnout, parenting, family systems, network science, systemic approach to family 


\section{Why We Should Move from Reductionism and Embrace a Network Approach to Parental Burnout}

"I think the next $\left[21^{\text {st }}\right]$ century will be the century of complexity"

- Stephen Hawking (2000)

For decades, science has attempted to understand the world by boiling concepts down to their simplest components, such as searching for a singular biological element or straightforward model to account for complex psychological processes (Barabási, 2012, 2016). Yet, this quest for reductionism has fallen short. Scientists from different fields have repeatedly discovered that complex systems work together to generate ecological, social, human, and biological processes, and that singular components or simple models cannot adequately explain these complex systems. There is now a move to map the world's complexities using a network approach, visualizing any system as a network to allow scientists to understand how a system's many components interact. This network takeover has only become possible in the last few decades, with growing computational power, and many fields have adopted this network science to map their systems of interest.

Clinical psychology research has started to embrace this perspective as well, conceptualizing psychological constructs as dynamic interacting networks of symptoms (for conceptual discussion, see Borsboom, 2017; Borsboom \& Cramer, 2013; for systematic reviews, see Contreras et al., 2019; Robinaugh et al., 2019). In this approach, a network depicts a mental disorder, with symptoms represented as "nodes," and the associations between symptoms represented as edges connecting them (see Figure 1B). This allows researchers to observe many symptoms simultaneously and investigate how they interact together and (reciprocally) influence one another. Beyond mere symptoms, if researchers are 
interested in how symptoms interact with relevant contextual variables (e.g., environmental variables such as daily life stressors) or individual variables (such as self-beliefs, emotion regulation processes, or neurocognitive mechanisms), they can represent these as nodes in the network as well (for a discussion, see Jones et al., 2017; for recent empirical examples, see Bernstein et al., 2020; Heeren et al., 2020; Kraft et al., 2019; Weiss et al., 2020). Adopting a network approach has theoretical implications for how we understand mental disorders. In a traditional latent model (see Figure 1A), mental disorders are viewed as latent (or underlying) variables that cause their symptoms (Borsboom, 2008). For example, the entity of "depression" would cause fatigue, sleep problems, loss of interest, and other symptoms, and these symptoms would not influence one another (Borsboom \& Cramer, 2013; Schmittmann et al., 2013). Indeed, when statistically modeling the relationship between a hypothesized latent variable and a set of its indicators, it is mathematically assumed that the observable indicators cannot be directly related — a phenomenon called the assumption of local/conditional independence (for further discussion and mathematical details, see Borsboom, 2008; Holland \& Rosenbaum, 1986; Junker \& Sijtsma, 2001; but see Bringmann \& Eronen, 2018 for a discussion on the blurred boundaries between latent and network models). However, in most cases, psychopathology symptoms not only interact (i.e., sleep problems leading to fatigue), but their interactions could be crucial to understanding how the disorder develops and maintains (e.g., if fatigue leads to depressed mood and loss of interest, which then lead to more sleep problems, this would be a feedback loop maintaining these symptoms; Borsboom \& Cramer, 2013). A network approach embraces these complex dynamics of mental disorders by focusing on symptoms and their interactions, allowing researchers to visualize all symptom interactions at once. For a visual contrast between a traditional latent approach and a network approach, see Figure 1. 
Capitalizing on tools developed in network science, researchers can also identify the central nodes (or most essential components) that drive a network system: central nodes are closely connected to the entire network ${ }^{1}$, and so can more easily influence other nodes (Borgatti, 2005; McNally, 2016; Opsahl et al., 2010). In psychopathology networks, this can have clinical implications. Treating a highly central node (such as through a targeted intervention) could beneficially cascade through the psychopathology network system, dampening the activation of other nodes (Fried et al., 2017; McNally, 2016). Yet, this conjecture rests on the assumption that one can deactivate a network's nodes in a specific, isolated fashion. It is possible that such specific targeting may be very difficult to achieve in practice (i.e., while aiming to target a given node, several nodes might be directly affected at once, not just the node of interest; for a discussion, see Valente, 2012). Nonetheless, a network approach invites promising new paradigms for clinical practice and treatment.

Network science can thus contribute to many fields, especially those that conceptualize their subject as a network without coordinated statistical tools; one key example involves family studies. The commonly termed "systemic" approach (e.g., Jackson, 1965; Minuchin, 1974; for an overview, see Dallos \& Draper, 2010) has theoretically prevailed for years in family research and therapy. This approach holds that to understand an individual's cognitions and behaviors also requires understanding the individual as embedded within a context and examining how that individual affects and responds to others in their family system (e.g., Bavelas \& Segal, 1982; Watzlawick et al., 1967). However, although

\footnotetext{
${ }^{1}$ There are a number of ways to define a node's centrality. The most common centrality indices for psychology research include strength (i.e., sum of the absolute edge weights that connect to that node), expected influence (similar but takes the positive or negative valence of the edge into account instead of the absolute value), closeness (i.e., that captures the average distance of a node from all other nodes in the network, and is computed from the inverse of the weighted sum of shortest path lengths connecting a given node to all the other nodes in the network), and betweenness (i.e., the number of times a node lies on the shortest path connecting two other nodes). For more information on centrality measures, see Bringmann et al., 2019; McNally, 2016; Opsahl et al., 2010).
} 
psychological theory and therapy (e.g., Sydow et al., 2013) have approached family processes as a dynamic network system (e.g., Bavelas \& Segal, 1982; Watzlawick et al., 1967), the ability to perform analogous statistical analyses remained limited. With the recent intense development in network science, though, there now exists both the computational capability and the mathematical tools to study the family as an actual dynamic network system.

Parental burnout is a prime candidate to represent and investigate using a network approach, as parental burnout is inextricable from its family system. Parental burnout involves emotional exhaustion, emotional distance from one's children, feeling fed up as a parent, and a sense of contrast with the previous ideal parental self (Roskam et al., 2018; for more indepth details, see Mikolajczak \& Roskam, 2020). The current research framework posits that parental burnout occurs when parents chronically lack the parenting resources needed to cope with their specific parenting-related stressors (Mikolajczak \& Roskam, 2018). What resources parents can access vary: from adequate financial resources to emotional resources, including social support, a cooperative co-parent, and sufficient leisure time (Mikolajczak et al., 2018). Parenting stressors can include anything from having multiple very young children, striving to be a perfect parent, or living with family disorganization (Mikolajczak et al., 2018). The particularities of each family and each parent combine to form their specific parenting risks and resources (which can vary culturally; Mikolajczak \& Roskam, 2018), and so by its very nature, parental burnout cannot be separated from its specific family context.

On a conceptual level, a network approach to parental burnout prioritizes the view that parental burnout emerges through the interactions of its constitutive elements (Schmittmann et al., 2013). This view dovetails with the foremost theoretical framework to explain parental burnout, which specifically emphasizes that a variety of family-specific factors (categorized as either "risks" or "resources," as mentioned above) combine over time and eventually result in a full-blown episode of parental burnout (Mikolajczak \& Roskam, 2018). Current research 
therefore frames the development and persistence of parental burnout as an accumulation of stressors without adequate resources to compensate, and thus rooted in the parent's immediate and extended context (e.g., their relationship with their spouse, their support network, their personality, their ability to regulate emotions and stress, the needs of their children, their financial situation). Therefore, a network model suits this theoretical framework of parental burnout exceptionally well, since network theory similarly posits that parental burnout arises through the interactions of its constitutive components (e.g., symptoms, family context, and external support). Moreover, although research into parental burnout is still in early stages (starting in earnest with Roskam et al., 2017), most research currently available assesses parental burnout as a unified latent construct. This loses valuable information by collapsing the different features of parental burnout into one sum score and ignoring any possibility that these distinct features could interact with the family system in different ways. Interestingly, practitioners know from experience that the different components of parental burnout interact with one another (e.g., the frequency of experiencing emotional exhaustion may increase the frequency of emotional distance, and vice versa; the frequency of feeling inefficient as a parent may foster emotional distance, and vice versa).

Not surprisingly, the only network analysis of parental burnout thus far published (Blanchard et al., 2020) supports this idea: parental burnout's features do interact in different ways with family-related variables. In this large cross-sectional network analysis $(n=1551)$, the authors (Blanchard et al., 2020) investigated the interactions between the three components of the parental burnout inventory (distance, exhaustion, and parental inefficacy; Roskam et al., 2017), as well as marital problems (specifically conflicts and estrangement) and child maltreatment (precisely neglect and violence). The study found that emotional distance was highly central, meaning it emerged as the most influential node in the network system. In particular, emotional distance was strongly connected with the other parental 
burnout features, but also with neglectful and violent behaviors toward children. These observations propose emotional distance as a key variable that maintains parental burnout within the family system, and that specifically seems tied to many of the negative consequences toward the children. In brief, this study demonstrates how a network approach can pinpoint which features of parental burnout interact with the detrimental consequences for the family system, while also generating hypotheses for how parental burnout might develop and persist.

This network study demonstrated the usefulness of investigating parental burnout within its family context and focusing on its components, but to confirm and further investigate how parental burnout operates within a family system requires investigating the temporal relationships between variables. Temporal networks can be used to assess and visualize how parental burnout components evolve over time within their family system. These networks include directed edges (i.e., connections between nodes where the direction of temporal influence is marked with an arrow; for an illustration, see Figure 1B), thus illustrating how variables impact each other over time, mapping one-way or reciprocal influences as well as multi-component feedback loops (Bringmann et al., 2013). Temporal networks have previously been used to investigate, among other things, direct and indirect connections between depression symptoms (Bringmann et al., 2015); to investigate the role daily stress plays in the frequency and persistence of psychotic experiences (Klippel et al., 2018); to examine how startle response, avoidance, blame, and negative emotions influence PTSD symptomatology at the next timepoint (Greene et al., 2018); and to disentangle how fatigue, loneliness, passive social media use, and depressive symptoms temporally influence one another (Aalbers et al., 2018).

Estimating temporal networks requires intensive time-series data such as experience sampling measurements (ESM), which involves assessing relevant variables once or multiple 
times a day over a long period of time (Palmier-Claus et al., 2011). This type of dense timeseries data would be particularly relevant when studying parenting, since the subjective experience of parenting may frequently change due to shifts in parents' and children's behavior and changing contextual factors. Time-series data also suits investigations into parental burnout, which is theorized to develop through a chronic imbalance between parenting risks and resources, worsening with time (Mikolajczak \& Roskam, 2018). Therefore, a network approach invites systemic questions about parental burnout: What are the central components in a time-lagged parental burnout network? What are the key feedback loops? Which family-related variables influence which parental burnout features, and viceversa? All of which distills into the fundamental dynamic questions: Why and how does parental burnout develop and persist?

A network perspective also offers intriguing opportunities to incorporate within clinical practice. For example, central nodes have been empirically linked with the prognosis of eating disorders (Elliott et al., 2019) and post-traumatic stress disorder (Papini et al., 2020). The mathematical notion of preferential attachment also supports that central nodes are key to how a network develops, positing that newly activated nodes connect to highly connected nodes first (Barabási \& Albert, 1999). Since central nodes are closely connected with many other nodes, researchers have theorized that turning off a central node (such as through a targeted intervention) could lead to a beneficial cascade, deactivating nodes throughout the entire network (McNally, 2016). Unfortunately, no experimental manipulations have yet tested this hypothesis (Robinaugh et al., 2019). Moreover, psychometricians advise caution when interpreting centrality metrics (Bringmann et al., 2019), but the clinical relevance of central nodes remains an intriguing topic for future research. To give an example relevant to parental burnout, if future research confirms that emotional distance is central to parental burnout networks, then clinicians could aim to specifically reduce parents' emotional distance 
toward their children, potentially through mental imagery exercises such as imagery rescripting (Blackwell, 2019; Holmes \& Mathews, 2010). Since we found that emotional distance was strongly connected to the other parental burnout features as well as child maltreatment (Blanchard et al., 2020), this targeted intervention should diminish these variables as well.

Another promising clinical tool are idiographic networks, which are temporal networks generated from ESM data for just one person, allowing both the patient and the therapist a visual, personalized window into the patient's daily experiences (Epskamp, van Borkulo, et al., 2018; for applied examples, see Bak et al., 2016; David et al., 2018). Thus, these personalized networks offer the potential to guide clinical practice by highlighting nodes that are central to that individual's network, as well as personalized clinical pathways that could be pursued during treatment (Epskamp, van Borkulo, et al., 2018). Although widespread adoption of personalized symptom networks in therapy is not immediately applicable, as they still require additional clinical validation and methodological refinements (Wichers et al., 2017), they offer great promise for the near future and are already the subject of serious research interest (Epskamp, van Borkulo, et al., 2018; Lydon-Staley et al., 2019; for an extended discussion of the clinical implications of such an individualized network approach, see David et al., 2018). We further foresee the development of individualized family network modeling, where a family records their feelings and behaviors over a period of time to generate a visual representation of their family dynamics. There currently exist advanced methods (using similar estimation techniques to network analyses) to examine emotion dynamics in pairs of people (Bringmann et al., 2018). Integrating these methods with network analyses and extending them to multiple people (e.g., a family) is a forthcoming possibility. We also anticipate the option of not only modeling one individual's network within their family context, but also modeling each individual within a family as well as 
modeling the interactions between family members, by layering individualized networks within a family network (Guloksuz et al., 2017; potential statistical tools include multiplex models; Shang, 2015). Individualized family networks would grant families and clinicians personalized insight into how their family system operates. This possibility would be helpful not only in informing parental burnout treatment but also more broadly in informing family systems theory and its applications in therapy.

Moreover, as noted by Borsboom and Cramer (2013), techniques from the study of sudden transitions in ecosystems (Hirota et al., 2011) may also help identify when a temporal network is on the brink of tipping into a disordered state or returning to a mentally healthy one (for an example with depression, see van de Leemput et al., 2014). One may thus envision adopting such techniques to identify when a family system is about to transition into a disordered state (e.g., parental burnout) or return to a healthy one, which could refine our understanding of resilience, recovery, and prevention on a family-wide level.

Many of these clinical implications require additional research and validation before they can be implemented. Therefore, we now outline some essential practical considerations and further resources for researchers who wish to conduct these further network analyses. The most straightforward way to investigate parental burnout and family variables from a network perspective is using cross-sectional data, such as from questionnaires. Cross-sectional networks are suited to examining many variables and their interactions at once, as well as identifying patterns or communities in the data (Guloksuz et al., 2017): they generate hypotheses that can be further investigated in temporal networks. These networks are usually built from a bivariate or partial correlation matrix; since these can include spurious relationships between nodes (represented as edges in the network), these networks are typically regularized to minimize false positive edges and yield a sparse network (Epskamp \& Fried, 2018). Network analyses can be applied to binary, ordinal, or continuous data (although 
the data might need to be transformed or alternate models used; for details, see Epskamp \& Fried, 2018). It is important to note that cross-sectional networks require many participants; power analyses are still rudimentary for network analyses (Epskamp, Borsboom, et al., 2018), but many researchers follow the rule of thumb from SEM to include at least 10 participants per parameter (Schreiber et al., 2006), meaning minimum a few hundred participants. Methods to gauge the robustness of network analyses do exist, however. Bootstrapping methods, for example, are typically used to estimate the stability of edge weights and centrality indices (for a tutorial, see Epskamp et al., 2018). Throughout the field of psychology, few replications of network studies have been conducted, so information is still lacking on the replicability and accuracy of network analyses. Thus, we urge researchers (including those conducting network analyses on parental burnout) to replicate network analyses, both by cross-validating within samples and by conducting identical analyses in different samples.

Temporal network analyses, for their part, require intensive time-series data (collected through methods such as ESM). Temporal network analyses therefore inherit the complexities of ESM data collection (Palmier-Claus et al., 2011). Examples of these complexities include determining the optimal distance between time points for the measured variables, constructing brief ESM items to accurately measure the desired variable in daily life with sufficient variability in participants' responses, and assessing the validity of the ESM items and determining whether (and how) these items should be combined in analyses. Different models exist to estimate temporal network analyses (for an overview, see Jordan et al., 2020), with the most common relying on multilevel vector autoregressive models (mlVAR; Bringmann et al., 2013), although new models to better handle the assumptions of temporal networks are continually being developed (e.g., Haslbeck et al., 2020). Temporal networks do not necessarily require large numbers of participants (particularly if the goal is to estimate an 
idiographic network), but they do require many timepoints. There are currently no standard methods to conduct power analyses for temporal networks (Jordan et al., 2020). However, an open-source simulation application was recently developed to help researchers estimate the number of timepoints their models require (Lafit et al., 2020). Only some temporal models include methods to assess their robustness (e.g., there are currently no bootstrapping routines developed for multilevel models; Jordan et al., 2020), and there are currently very few replications. We hope that additional replications and methods to assess model accuracy and robustness will be forthcoming.

In conclusion, a network approach to parental burnout focuses on the features of parental burnout as embedded within a specific family system and encourages dynamic investigations using intensive time-series data. Reducing parental burnout to one isolated syndrome - as done by most current analyses — does not fit the theoretical framework of parental burnout, largely ignores the specific family system, and neglects how parental burnout features interact with contextual family factors. We therefore urge researchers (and, with the gradual clinical applications of networks, practitioners) to adopt a network approach to parental burnout, particularly when their research question involves the family context or the specific, interactive, or reciprocal influences of the different features of parental burnout. We believe that this perspective can lead to more nuanced insights regarding how parental burnout affects the entire family, with all its contextual specificities. Although adopting a network approach can involve learning new analysis techniques, numerous resources exist to introduce and explain the concepts and analyses of network analyses, from simple online applications and open software (see http://psychosystems.org/software/) to R code tutorials (e.g., Epskamp, Borsboom, et al., 2018; Epskamp \& Fried, 2018; Jones et al., 2018). Hopefully, with continuing research on how network analyses can inform psychological 
treatment and targeted network interventions, intuitive tools, and clear guides for practitioners will also be freely developed and widely disseminated. 


\section{Acknowledgments}

Both authors are supported by a Coordinated Research Grant ("BParent") from the French Community of Belgium (ARC Grant n¹9/24-100). Prof. Alexandre Heeren is also supported (as FRS-FNRS research associate) by the FRS-FNRS Belgian National Science Foundation. These funds did not exert any influence or censorship of any kind on the present work. 


\section{References}

Aalbers, G., McNally, R. J., Heeren, A., de Wit, S., \& Fried, E. I. (2018). Social media and depression symptoms: A network perspective. Journal of Experimental Psychology: General. https://doi.org/10.1037/xge0000528

Bak, M., Drukker, M., Hasmi, L., \& Van Jim, O. S. (2016). An n=1 Clinical network analysis of symptoms and treatment in psychosis. PLOS ONE, 11(9), 1-15. https://doi.org/10.1371/journal.pone.0162811

Barabási, A. L. (2012). The network takeover. Nature Physics, 8(1), 14-16. https://doi.org/10.1038/nphys2188

Barabási, A. L. (2016). Network science. Cambridge university press. http://networksciencebook.com/

Barabási, A. L., \& Albert, R. (1999). Emergence of scaling in random networks. Science, 286, $509-512$.

Bavelas, J. B., \& Segal, L. (1982). Family systems theory: Background and implications. Journal of Communication, 32(3), 99-107.

Bernstein, E. E., Heeren, A., \& McNally, R. J. (2020). A network approach to understanding the emotion regulation benefits of aerobic exercise. Cognitive Therapy and Research, 44(1), 52-60. https://doi.org/10.1007/s10608-019-10039-6

Blackwell, S. E. (2019). Mental imagery: From basic research to clinical practice. Journal of Psychotherapy Integration, 29(3), 235-247. https://doi.org/10.1037/int0000108

Blanchard, M. A., Roskam, I., Mikolajczak, M., \& Heeren, A. (2020). A network approach to parental burnout [Preprint]. https://doi.org/10.31234/osf.io/swqfz

Borgatti, S. P. (2005). Centrality and network flow. Social Networks, 27(1), 55-71. https://doi.org/10.1016/j.socnet.2004.11.008 
Borsboom, D. (2008). Latent variable theory. Measurement: Interdisciplinary Research \& Perspective, 6(1-2), 25-53. https://doi.org/10.1080/15366360802035497

Borsboom, D. (2017). A network theory of mental disorders. World Psychiatry, 16(1), 5-13. https://doi.org/10.1002/wps.20375

Borsboom, D., \& Cramer, O. J. (2013). Network analysis: An integrative approach to the structure of psychopathology. Annual Review of Clinical Psychology, 9, 91-121. https://doi.org/10.1146/annurev-clinpsy-050212-185608

Bringmann, L. F., Elmer, T., Epskamp, S., Krause, R. W., Schoch, D., Wichers, M., Wigman, J. T. W., \& Snippe, E. (2019). What do centrality measures measure in psychological networks? Journal of Abnormal Psychology, 128(8), 892-903. https://doi.org/10.1037/abn0000446

Bringmann, L. F., \& Eronen, M. I. (2018). Don't blame the model: Reconsidering the network approach to psychopathology. Psychological Review, 125(4), 606-615. http://dx.doi.org/10.1037/rev0000108

Bringmann, L. F., Ferrer, E., Hamaker, E. L., Borsboom, D., \& Tuerlinckx, F. (2018). Modeling Nonstationary Emotion Dynamics in Dyads using a Time-Varying VectorAutoregressive Model. Multivariate Behavioral Research, 53(3), 293-314. https://doi.org/10.1080/00273171.2018.1439722

Bringmann, L. F., Lemmens, L. H. J. M., Huibers, M. J. H., Borsboom, D., \& Tuerlinckx, F. (2015). Revealing the dynamic network structure of the Beck Depression Inventory-II. Psychological Medicine, 45(4), 747-757. https://doi.org/10.1017/S0033291714001809

Bringmann, L. F., Vissers, N., Wichers, M., Geschwind, N., Kuppens, P., Peeters, F., Borsboom, D., \& Tuerlinckx, F. (2013). A network approach to psychopathology: 
New insights into clinical longitudinal data. PLoS ONE, 8(4). https://doi.org/10.1371/journal.pone.0060188

Contreras, A., Nieto, I., Valiente, C., Espinosa, R., \& Vazquez, C. (2019). The study of psychopathology from the network analysis perspective: A systematic review. Psychotherapy and Psychosomatics, 88(2), 71-83. https://doi.org/10.1159/000497425

Dallos, R., \& Draper, R. (2010). An Introduction To Family Therapy: Systemic Theory and Practice. McGraw-Hill Education (UK).

David, S. J., Marshall, A. J., Evanovich, E. K., \& Mumma, G. H. (2018). Intraindividual Dynamic Network Analysis - Implications for Clinical Assessment. Journal of Psychopathology and Behavioral Assessment, 40(2), 235-248. https://doi.org/10.1007/s10862-017-9632-8

Elliott, H., Jones, P. J., \& Schmidt, U. (2019). Central symptoms predict post-treatment outcomes and clinical impairment in anorexia nervosa: A network analysis. Clinical Psychological Science, 1-16. https://doi.org/10.1177/2167702619865958

Epskamp, S., Borsboom, D., \& Fried, E. I. (2018). Estimating psychological networks and their accuracy: A tutorial paper. Behavior Research Methods, 50(1), 195-212. https://doi.org/10.3758/s13428-017-0862-1

Epskamp, S., \& Fried, E. I. (2018). A tutorial on regularized partial correlation networks. Psychological Methods, 23(4), 617-634. https://doi.org/10.1037/met0000167

Epskamp, S., van Borkulo, C. D., van der Veen, D. C., Servaas, M. N., Isvoranu, A. M., Riese, H., \& Cramer, A. O. J. (2018). Personalized network modeling in psychopathology: The importance of contemporaneous and temporal connections. Clinical Psychological Science, 6(3), 416-427. https://doi.org/10.1177/2167702617744325 
Fried, E. I., van Borkulo, C. D., Cramer, A. O. J., Boschloo, L., Schoevers, R. A., \& Borsboom, D. (2017). Mental disorders as networks of problems: A review of recent insights. Social Psychiatry and Psychiatric Epidemiology, 52(1), 1-10. https://doi.org/10.1007/s00127-016-1319-z

Greene, T., Gelkopf, M., Epskamp, S., \& Fried, E. (2018). Dynamic networks of PTSD symptoms during conflict. Psychological Medicine, 48(14), 2409-2417. https://doi.org/10.1017/S0033291718000351

Guloksuz, S., Pries, L. K., \& Van Os, J. (2017). Application of network methods for understanding mental disorders: Pitfalls and promise. Psychological Medicine, 47(16), 2743-2752. https://doi.org/10.1017/S0033291717001350

Haslbeck, J. M. B., Bringmann, L. F., \& Waldorp, L. J. (2020). A Tutorial on Estimating Time-Varying Vector Autoregressive Models. Multivariate Behavioral Research, $O(0), 1-30$. https://doi.org/10.1080/00273171.2020.1743630

Hawking, S. (2000, January 20). The "millenium” interview. San Jose Mercury News.

Heeren, A., Bernstein, E. E., \& McNally, R. J. (2020). Bridging maladaptive social selfbeliefs and social anxiety: A network perspective. Journal of Anxiety Disorders, 74, 102267. https://doi.org/10.1016/j.janxdis.2020.102267

Hirota, M., Holmgren, M., Nes, E. H. V., \& Scheffer, M. (2011). Global Resilience of Tropical Forest and Savanna to Critical Transitions. Science, 334(6053), 232-235. https://doi.org/10.1126/science. 1210657

Holland, P. W., \& Rosenbaum, P. R. (1986). Conditional association and unidimensionality in monotone latent variable models. The Annals of Statistics, 14(4), 1523-1543. JSTOR.

Holmes, E. A., \& Mathews, A. (2010). Mental imagery in emotion and emotional disorders. Clinical Psychology Review, 30, 349-362. https://doi.org/10.1016/j.cpr.2010.01.001 Jackson, D. (1965). The Study of Family. Family Process, 4, 1-20. 
Jones, P. J., Heeren, A., \& McNally, R. J. (2017). Commentary: A network theory of mental disorders. Frontiers in Psychology, 8, 1305. https://doi.org/10.1002/wps.20375

Jones, P. J., Mair, P., \& McNally, R. J. (2018). Visualizing Psychological Networks: A Tutorial in R. Frontiers in Psychology, 9. https://doi.org/10.3389/fpsyg.2018.01742

Jordan, D. G., Winer, E. S., \& Salem, T. (2020). The current status of temporal network analysis for clinical science: Considerations as the paradigm shifts? Journal of Clinical Psychology. https://doi.org/10.1002/jclp.22957

Junker, B. W., \& Sijtsma, K. (2001). Cognitive Assessment Models with Few Assumptions, and Connections with Nonparametric Item Response Theory. Applied Psychological Measurement, 25(3), 258-272. https://doi.org/10.1177/01466210122032064

Klippel, A., Viechtbauer, W., Reininghaus, U., Wigman, J., van Borkulo, C., Myin-Germeys, I., \& Wichers, M. (2018). The cascade of stress: A network approach to explore differential dynamics in populations varying in risk for psychosis. Schizophrenia Bulletin, 44(2), 328-337. https://doi.org/10.1093/schbul/sbx037

Kraft, B., Jonassen, R., Heeren, A., Harmer, C., Stiles, T., \& Landrø, N. I. (2019). Attention bias modification in remitted depression is associated with increased interest and leads to reduced adverse impact of anxiety symptoms and negative cognition. Clinical Psychological Science, 7(3), 530-544. https://doi.org/10.1177/2167702618822480

Lafit, G., Adolf, J., Dejonckheere, E., Myin-Germeys, I., Viechtbauer, W., \& Ceulemans, E. (2020). Selection of the number of participants in intensive longitudinal studies: A user-friendly Shiny app and tutorial to perform power analysis [Preprint]. PsyArXiv. https://doi.org/10.31234/osf.io/dq6ky

Lydon-Staley, D. M., Barnett, I., Satterthwaite, T. D., \& Bassett, D. S. (2019). Digital phenotyping for psychiatry: Accommodating data and theory with network science 
methodologies. Current Opinion in Biomedical Engineering, 9, 8-13. https://doi.org/10.1016/j.cobme.2018.12.003

McNally, R. J. (2016). Can network analysis transform psychopathology? Behaviour Research and Therapy, 86, 95-104. https://doi.org/10.1016/j.brat.2016.06.006

Mikolajczak, M., Raes, M. E., Avalosse, H., \& Roskam, I. (2018). Exhausted Parents: Sociodemographic, Child-Related, Parent-Related, Parenting and Family-Functioning Correlates of Parental Burnout. Journal of Child and Family Studies, 27(2), 602-614. https://doi.org/10.1007/s10826-017-0892-4

Mikolajczak, M., \& Roskam, I. (2018). A theoretical and clinical framework for parental burnout: The balance between risks and resources (BR2). Frontiers in Psychology, 9, 886. https://doi.org/10.3389/fpsyg.2018.00886

Mikolajczak, M., \& Roskam, I. (2020). [From this issue; Title to be updated during proof editing].

Minuchin, S. (1974). Families and Family Therapy. Harvard University Press.

Opsahl, T., Agneessens, F., \& Skvoretz, J. (2010). Node centrality in weighted networks: Generalizing degree and shortest paths. Social Networks, 32(3), 245-251. https://doi.org/10.1016/j.socnet.2010.03.006

Palmier-Claus, J. E., Myin-Germeys, I., Barkus, E., Bentley, L., Udachina, A., Delespaul, P. a. E. G., Lewis, S. W., \& Dunn, G. (2011). Experience sampling research in individuals with mental illness: Reflections and guidance. Acta Psychiatrica Scandinavica, 123(1), 12-20. https://doi.org/10.1111/j.1600-0447.2010.01596.x

Papini, S., Rubin, M., Telch, M. J., Smits, J. A. J., \& Hien, D. A. (2020). Pretreatment Posttraumatic Stress Disorder Symptom Network Metrics Predict the Strength of the Association Between Node Change and Network Change During Treatment. Journal of Traumatic Stress, 33(1), 64-71. https://doi.org/10.1002/jts.22379 
Robinaugh, D. J., Hoekstra, R. H. A., Toner, E. R., \& Borsboom, D. (2019). The network approach to psychopathology: A review of the literature $2008-2018$ and an agenda for future research. Psychological Medicine, 50(3), 1-14. https:// doi.org/10.1017/S0033291719003404

Roskam, I., Brianda, M. E., \& Mikolajczak, M. (2018). A step forward in the conceptualization and measurement of parental burnout: The Parental Burnout Assessment (PBA). Frontiers in Psychology, 9, 758. https://doi.org/10.3389/fpsyg.2018.00758

Roskam, I., Raes, M. E., \& Mikolajczak, M. (2017). Exhausted parents: Development and preliminary validation of the parental burnout inventory. Frontiers in Psychology, 8 , 163. https://doi.org/10.3389/fpsyg.2017.00163

Schmittmann, V. D., Cramer, A. O. J., Waldorp, L. J., Epskamp, S., Kievit, R. A., \& Borsboom, D. (2013). Deconstructing the construct: A network perspective on psychological phenomena. New Ideas in Psychology, 31(1), 43-53. https://doi.org/10.1016/j.newideapsych.2011.02.007

Schreiber, J. B., Nora, A., Stage, F. K., Barlow, E. A., \& King, J. (2006). Reporting structural equation modeling and confirmatory factor analysis results: A review. The Journal of Educational Research, 99(6), 323-338. https://doi.org/10.3200/JOER.99.6.323-338

Shang, Y. (2015). Deffuant model of opinion formation in one-dimensional multiplex networks. Journal of Physics A: Mathematical and Theoretical, 48(39), 395101. https://doi.org/10.1088/1751-8113/48/39/395101

Sydow, K. von, Retzlaff, R., Beher, S., Haun, M. W., \& Schweitzer, J. (2013). The Efficacy of Systemic Therapy for Childhood and Adolescent Externalizing Disorders: A Systematic Review of 47 RCT. Family Process, 52(4), 576-618. https://doi.org/10.1111/famp.12047 
Valente, T. W. (2012). Network interventions. Science, 336(6090), 49-53. https://doi.org/10.1126/science. 1217330

van de Leemput, I. A., Wichers, M., Cramer, A. O. J., Borsboom, D., Tuerlinckx, F., Kuppens, P., Van Nes, E. H., Viechtbauer, W., Giltay, E. J., Aggen, S. H., Derom, C., Jacobs, N., Kendler, K. S., Van Der Maas, H. L. J., Neale, M. C., Peeters, F., Thiery, E., Zachar, P., \& Scheffer, M. (2014). Critical slowing down as early warning for the onset and termination of depression. Proceedings of the National Academy of Sciences of the United States of America, 111(1), 87-92. https://doi.org/10.1073/pnas.1312114110

Watzlawick, P., Bavelas, J. B., \& Jackson, D. D. (1967). Pragmatics of Human Communication: A Study of Interactional Patterns, Pathologies and Paradoxes. Norton.

Weiss, N. H., Contractor, A. A., Raudales, A. M., Greene, T., \& Short, N. A. (2020). Extending our understanding of the association between posttraumatic stress disorder and positive emotion dysregulation: A network analysis approach. Journal of Anxiety Disorders, 71, 102198. https://doi.org/10.1016/j.janxdis.2020.102198

Wichers, M., Wigman, J. T. W., Bringmann, L. F., \& de Jonge, P. (2017). Mental disorders as networks: Some cautionary reflections on a promising approach. Social Psychiatry and Psychiatric Epidemiology, 52(2), 143-145. https://doi.org/10.1007/s00127-016$1335-\mathrm{z}$ 


\section{Figure 1}

Comparing Latent vs. Network Approaches to Mental Disorders

A) Latent Approach

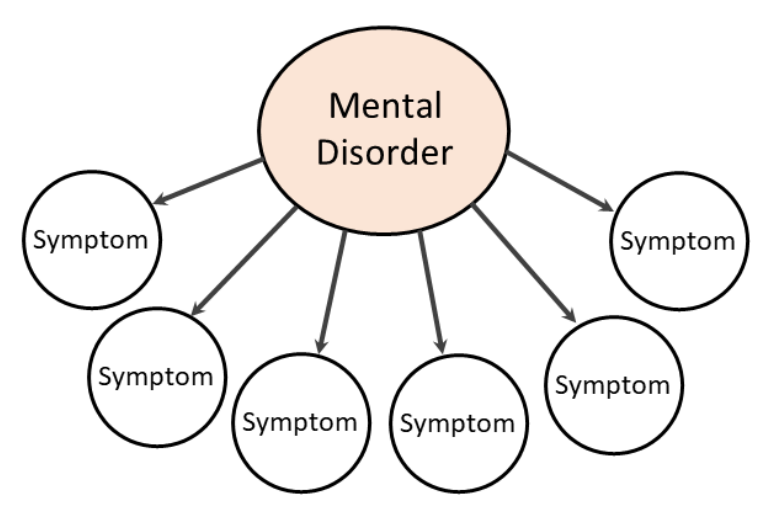

B) Network Approach

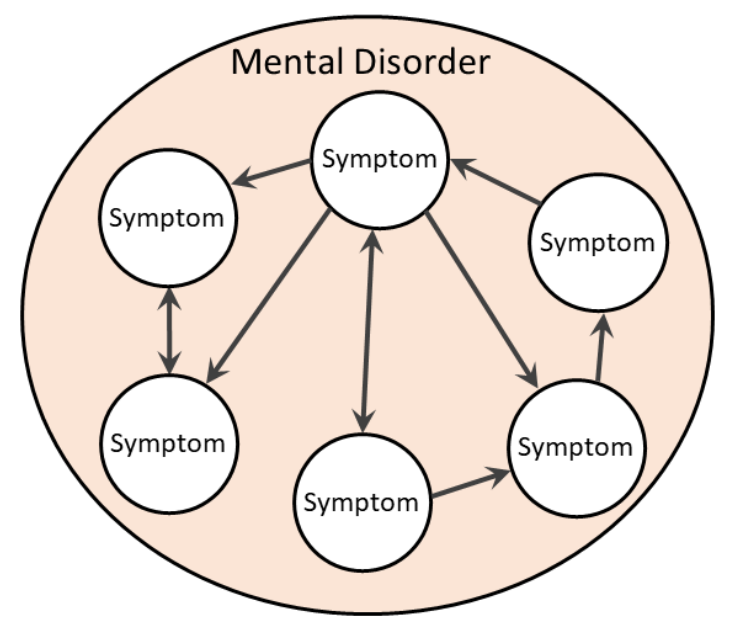

Note. Panel A: When representing a mental disorder from a traditional latent approach, the mental disorder is conceptualized as a latent variable that causes all of it symptoms, and the symptoms usually do not influence each other. Panel B: When representing a mental disorder from a network approach, the mental disorder is a network made up of its symptoms: each symptom is a node, and the associations between symptoms are represented as edges (lines). In this representation, the edges are directed, with an arrow signifying the direction of influence between nodes. Inspired from "Commentary: A Network Theory of Mental Disorders,” by P.J. Jones, A. Heeren. \& R. McNally, 2017, Frontiers in Psychology, 8, p. 2. 